\title{
OBSERVING GRBS WITH THE LOFT WIDE FIELD MONITOR
}

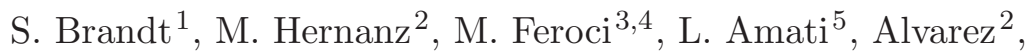
P. Azzarello ${ }^{6}$, D. Barret ${ }^{7}$, E. Bozzo ${ }^{6}$, C. Budtz-Jørgensen ${ }^{1}$, R. Campana ${ }^{3,4}$, A. Castro-Tirado ${ }^{8}$, A. $\operatorname{Cros}^{7}$, E. Del Monte ${ }^{3,4}$, I. Donnarumma ${ }^{3}$, Y. Evangelista ${ }^{3,4}$, J.L. Galvez Sanchez ${ }^{2}$, D. Götz ${ }^{9}$, F. Hansen ${ }^{1}$, J.W. den Herder ${ }^{10}$, A. Hornstrup ${ }^{1}$, R. Hudec ${ }^{11,12}$, D. Karelin ${ }^{2}$, M. van der Klis ${ }^{13}$, S. Korpela ${ }^{14}$, I. Kuvvetli ${ }^{1}$, N. Lund ${ }^{1}$, P. Orleanski ${ }^{15}$, M. Pohl ${ }^{16}$, A. Rachevski ${ }^{17}$, A. Santangelo ${ }^{18}$, S. Schanne ${ }^{9}$, C. Schmid ${ }^{19}$, L. Stella ${ }^{20}$, S. Suchy ${ }^{18}$, C. Tenzer ${ }^{18}$, A. Vacchi ${ }^{17}$, J. Wilms ${ }^{19}$, N. Zampa ${ }^{17}$, J.J.M. in't Zand ${ }^{10}$ and A. Zdziarski ${ }^{21}$

\footnotetext{
1 National Space Institute, Technical University of Denmark, Elektrovej Bld 327, 2800 Kgs Lyngby, Denmark

2 IEEC-CSIC-UPC-UB, Carrer del Gran Capità, 2, 08034 Barcelona, Spain

3 IAPS-INAF, via del Fosso del Cavaliere 100, 00133 Rome, Italy

${ }^{4}$ INFN, Sez. Roma Tor Vergata, via della Ricerca Scientifica 1, 00133 Rome, Italy

5 INAF-IASF-Bologna, via P. Gobetti, 101, 40129 Bologna, Italy

6 ISDC, Geneve University, Chemin d'Ecogia 16, 1290 Versoix, Switzerland

7 IRAP, avenue du Colonel Roche, 9, BP. 44346, Toulouse, France

8 Instituto Astrofisica de Andalucia, Glorieta de la Astronomía, s/n., 18008 Granada, Spain

9 CEA Saclay, DSM/IRFU/SAp, 91191 Gif-sur-Yvette, France

10 SRON, Sorbonnelaan 2, 3584 CA Utrecht, The Netherlands

11 Astronomical Institute of the Academy of Sciences of the Czech Republic, Fricova 298, 25165 Ondrejov, Czech Republic

12 Czech Technical University in Prague, Zikova 1903/4, 16636 Praha 6, Czech Republic

13 Astronomical Institute Anton Pannekoek, University of Amsterdam, Science Park 904, 1098 XH Amsterdam, The Netherlands

14 Department of Physics, Division of Geophysics and Astronomy, PO Box 48, 00014 University of Helsinki, Finland

15 Space Research Centre, Warsaw, Bartycka 18A, Warszawa, Poland

16 DPNC, Geneve University, Quai Ernest-Ansermet 30, 1205 Geneva, Switzerland

17 INFN, Trieste, via A. Valerio 2, 34127 Trieste, Italy

18 IAAT University of Tuebingen, Sand 1, 72076 Tuebingen, Germany

19 University of Erlangen-Nuremberg, Schlossplatz 4, 91054 Erlangen, Germany

20 INAF-OA Roma, via Frascati, 33, 00040 Monte Porzio Catone, Italy

21 Copernicus Astronomical Center, Bartycka 18, Warsaw, Poland
} 


\begin{abstract}
LOFT (Large Observatory For X-ray Timing) is one of the four candidate missions currently under assessment study for the M3 mission in ESAs Cosmic Vision program to be launched in 2024. LOFT will carry two instruments with prime sensitivity in the $2-30 \mathrm{keV}$ range: a $10 \mathrm{~m}^{2}$ class large area detector (LAD) with a $<1^{\circ}$ collimated field of view and a wide field monitor (WFM) instrument. The WFM is based on the coded mask principle, and 5 camera units will provide coverage of more than $1 / 3$ of the sky. The prime goal of the WFM is to detect transient sources to be observed by the LAD. With its wide field of view and good energy resolution of $<500 \mathrm{eV}$, the WFM will be an excellent instrument for detecting and studying GRBs and X-ray flashes. The WFM will be able to detect $\sim 150$ gamma ray bursts per year, and a burst alert system will enable the distribution of $\sim 100$ GRB positions per year with $\mathrm{a} \sim 1$ arcmin location accuracy within $30 \mathrm{~s}$ of the burst.
\end{abstract}

\title{
1 Introduction
}

LOFT (Large Observatory For X-ray Timing) [1,2], is one of the four missions selected in 2011 for assessment study for the ESA M3 mission [3]. The final mission selection will be done in early 2014. LOFT will, if selected, carry two science instruments, both based on Silicon drift detectors (SDDs), with a primary energy range of 2-30 keV. A schematic view of the LOFT spacecraft and payload is shown in Figure 1.

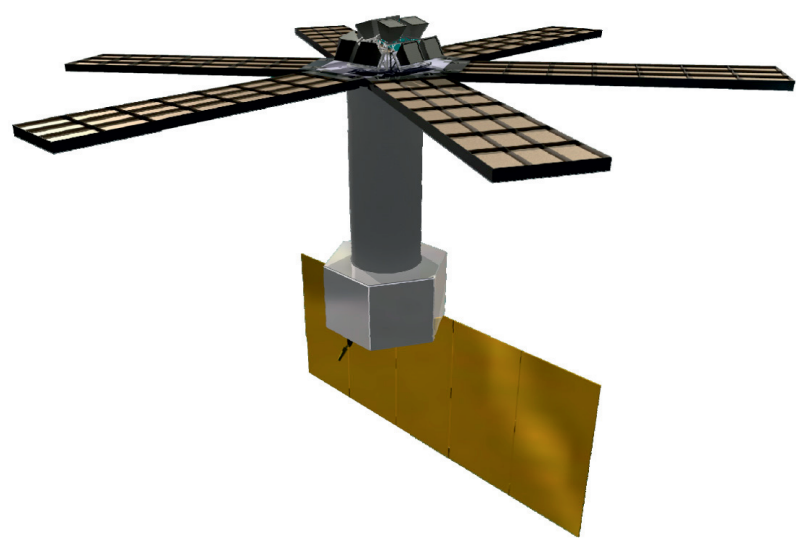

Fig. 1. Schematic views of the LOFT spacecraft showing the deployed Large Area Detector (LAD) panels attached to the optical bench. The Wide Field Monitor (WFM) is placed on the optical bench at the top. The direction of maximum response of the WFM is co-aligned with the viewing direction of the LAD.

The Large Area Detector (LAD) is a collimated instrument with an effective area of $\sim 10 \mathrm{~m}^{2}$ designed for X-ray timing with a better than $250 \mathrm{eV}$ energy 
resolution and a $10 \mu \mathrm{s}$ time resolution [4]. The second instrument on LOFT is a Wide Field Monitor (WFM) based on the coded mask principle, and with a detector plane based on Silicon Drift Detectors, much similar to the LAD detectors, but with a design optimized for position determination of the incoming X-rays [5]. The WFM is primarily needed to detect interesting targets for the LAD, but with exciting and unique capabilities on its own. In order to optimize for the detection of weak sources in a background dominated by cosmic diffuse emission and other $\mathrm{X}$-ray sources the coded mask has a $25 \%$ open fraction. We note that for GRBs an open fraction of $50 \%$ is normally used, when the signal is dominating the background. A single WFM camera and the full WFM camera assembly on the spacecraft is shown in Figure 2.

The mission duration will be 4 years, and is mainly driven by the statistics of the occurrence of the bright black hole transients, which are a class of prime targets for the LAD. The orbit for LOFT is nearly equatorial with an altitude of $\sim 550 \mathrm{~km}$.
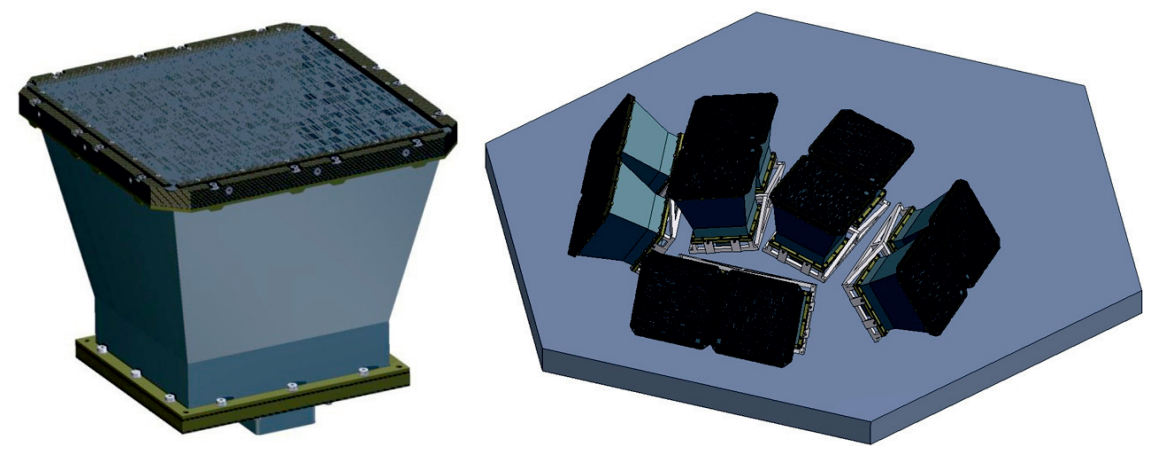

Fig. 2. Left: one WFM coded mask camera. The angular resolution is highly asymmetric at $5^{\prime} \times 5^{\circ}$. A pair of cameras oriented at a relative rotation of $90^{\circ}$ offers a $5^{\prime} \times 5^{\prime}$ combined resolution and a source position accuracy of $1^{\prime} \times 1^{\prime}$. Right: the WFM assembly mounted on the LOFT optical bench consisting of 5 co-aligned camera pairs, for a total of 10 cameras.

\section{The WFM scince goals}

The main goal for the WFM is to detect new transients and state changes of known sources suitable for observation with the LAD. However, the WFM will be able to do important science on its own. With a very large field of view, covering more than $1 / 3$ of the sky, the WFM offers a high duty cycle compared to other X-ray monitors with a scanning mode of operation, like the past RXTE/ASM and the current MAXI monitor.

The LOFT-WFM is ideally suited to study the physics of prompt, explosive events, such as gamma-ray bursts, bursts and intermediate flares from magnetars, and X-ray bursts. The low-energy threshold at $2 \mathrm{keV}[1,2]$ is well below that of 
current and foreseen monitors such as Swift-BAT, and SVOM or UFFO and the energy resolution $<500 \mathrm{eV}$ (and a goal of $<300 \mathrm{eV}$ ) is unique. The soft response and good energy resolution of the WFM will allow the detection of spectral features in the soft prompt X-ray emission, where observations are still very limited. The source location accuracy is better than 1 arcmin for burst with sufficient counting statistics.

Table 1. Summary of the performance parameters of the LOFT WFM. The energy resolution is required to be better than $500 \mathrm{eV}$, but the goal is to achieve $300 \mathrm{eV}$ or better.

\begin{tabular}{|l|c|c|c|}
\hline Parameter & One Camera & $\begin{array}{c}\text { One Camera Unit } \\
=\mathbf{2} \text { crossed } \\
\text { cameras }\end{array}$ & Overall WFM \\
\hline Energy Range & $2-30(30-80) \mathrm{keV}$ & $2-30(30-80) \mathrm{keV}$ & $2-30(30-80) \mathrm{keV}$ \\
\hline Active Detector Area & $182 \mathrm{~cm}^{2}$ & $364 \mathrm{~cm}^{2}$ & $1820 \mathrm{~cm}^{2}$ \\
\hline $\begin{array}{l}\text { Peak Effective Area } \\
\text { (on-axis, through 25\% } \\
\text { open mask) }\end{array}$ & $>40 \mathrm{~cm}^{2}$ & $>80 \mathrm{~cm}^{2}$ & $>80 \mathrm{~cm}^{2}$ \\
\hline $\begin{array}{l}\text { Energy Resolution } \\
\text { FWHM }\end{array}$ & $<500 \mathrm{eV}$ & $<500 \mathrm{eV}$ & $<500 \mathrm{eV}$ \\
\hline $\begin{array}{l}\text { Field of View at Zero } \\
\text { Response }\end{array}$ & $90^{\circ} \times 90^{\circ}$ & $90^{\circ} \times 90^{\circ}$ & $210^{\circ} \times 90^{\circ}+45^{\circ} \times 90^{\circ}$ \\
\hline $\begin{array}{l}\text { Field of view at 20\% } \\
\text { response }\end{array}$ & $60^{\circ} \times 60^{\circ}$ & $60^{\circ} \times 60^{\circ}$ & $180^{\circ} \times 60^{\circ}+60^{\circ} \times 60^{\circ}$ \\
\hline Angular Resolution & $5^{\prime} \times 5^{\circ}$ & $5^{\prime} \times 5^{\prime}$ & $5^{\prime} \times 5^{\prime}$ \\
\hline $\begin{array}{l}\text { Point Source Location } \\
\text { Accuracy }(10 \sigma \text { signal })\end{array}$ & $<1^{\prime} \times 30^{\prime}$ & $<1^{\prime} \times 1^{\prime}$ & $270 \mathrm{mCrab}^{\prime}$ \\
\hline $\begin{array}{l}\text { On-axis sensitivity at } \\
5 \sigma \text { in 3 s, in Galactic } \\
\text { Center }\end{array}$ & $380 \mathrm{mCrab}$ & $270 \mathrm{mCrab}$ & $2.1 \mathrm{mCrab}^{\prime}$ \\
\hline $\begin{array}{l}\text { On-axis sensitivity at } \\
5 \sigma \text { in } 58 \text { ks } \\
(1 \text { day Galactic Center } \\
\text { pointing) }\end{array}$ & $3.0 \mathrm{mCrab}$ & $2.1 \mathrm{mCrab}$ & \\
\hline
\end{tabular}

Our simulations show that in the first year of the mission, LOFT-WFM will detect more than 50 gamma-ray bursts at redshifts $>2$ and the LOFT WFM will be able to contribute to the study of high z GRBs. Over a four-year mission, even conservative models predict that WFM will detect $15-30$ GRB at $\mathrm{z}>5$ and $\sim 5-10$ GRB at $\mathrm{z}>6$, which will be promptly localized and followed-up in multi-wavelength campaigns. These discoveries may shed light on fundamental topics such as the formation of population III stars, the star formation rate and the evolution of the interstellar medium of galaxies from the epoch of re-ionization.

We emphasize that no past, present, or planned GRB experiment has such a combination of low energy threshold, energy resolution, and wide field of view as the LOFT WFM. 


\section{The WFM sky coverage}

The WFM will be placed on the optical bench on top of the spacecraft. The WFM configuration consisting of 5 camera pairs (a total of 10 cameras) each with a field of view of $90^{\circ} \times 90^{\circ}$ at zero response. The WFM baseline configuration is illustrated in Figure 2. This configuration will cover $\sim 5.5$ steradian or $\sim 44 \%$ of the sky at zero response, and $\sim 4.2$ steradian or $1 / 3$ of the sky at $20 \%$ response relative to on-axis.

Four of the five units are arranged such that their viewing axes lies in the plane of the solar panel of the LOFT spacecraft, and the fifth unit is tilted out of this plane, away from the Sun, by $60^{\circ}$. The viewing directions of the four units are off-set by $\pm 15^{\circ}$ and $\pm 60^{\circ}$ relative to the LAD pointing direction, which also lies in the solar panel plane (see Fig. 1). The effective area of the full WFM assembly is shown in the right hand panel of Figure 3. With this arrangement, the two central units have the LAD target in their field, where the detectors are fully illuminated, providing the maximum WFM coverage, with $\sim 160 \mathrm{~cm}^{2}$ effective area. The zero response field of view of the 4 units is $210^{\circ} \times 90^{\circ}$. However, depending on the configuration of the LAD panels and the placement of the WFM units on the optical bench, only an unobstructed field of view of $180^{\circ} \times 90^{\circ}$ can be assured. The $60^{\circ}$ tilt of the two outer units with respect to the LAD pointing direction is preferred in order to have a reasonable response at the edge defined by the plane of the optical bench. In this configuration, the WFM nominally covers half of the sky that is accessible to LAD pointings. The WFM may therefore in just 2 LOFT pointings cover all the sky accessible to the LAD, or an area corresponding to at least $75 \%$ of the sky.
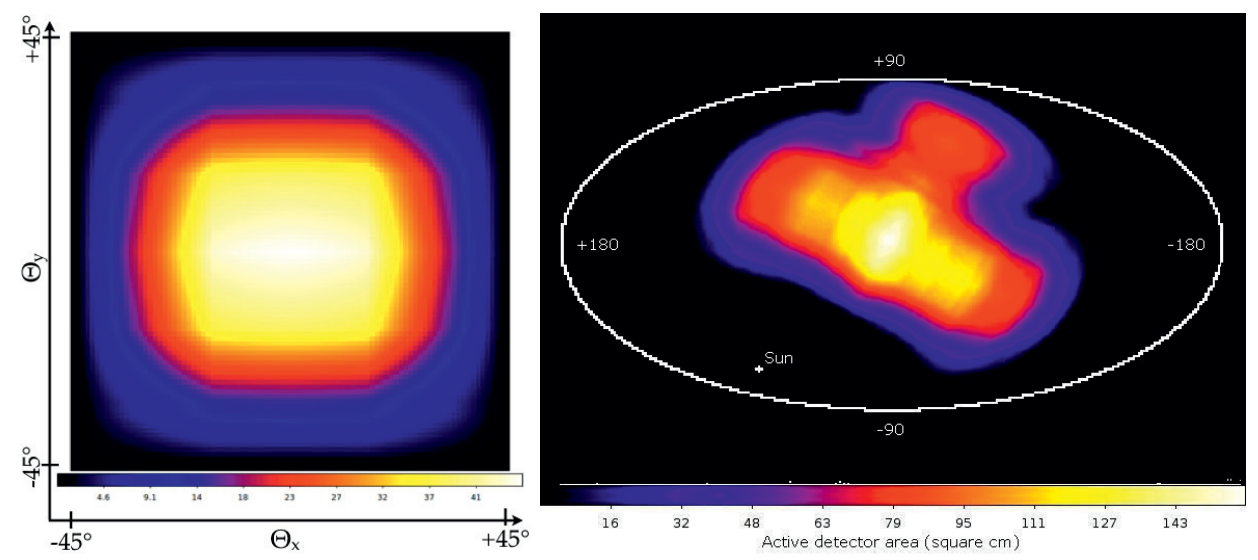

Fig. 3. Left panel: map of the single camera sensitive area expressed in $\mathrm{cm}^{2}$, with a maximum of $\sim 45 \mathrm{~cm}^{2}$ of a single camera. Right panel: map in Galactic coordinates of the active detector area for an example observation of the Galactic center. The effective area has its maximum of $\sim 160 \mathrm{~cm}^{2}$ in the direction of the LAD pointing. 


\section{The LOFT burst alert system}

The WFM is estimated to be able to detect $\sim 150$ Gamma Ray Bursts per year and we expect to be able to distribute near real time positions for $\sim 100$ burst per year. Scientifically it is highly desirable to do follow-up observations of these sources with other telescopes and instruments as soon as possible after (or even during) the event. Therefore LOFT will employ a VHF transmission capability to send a short message about the occurrence of such events with minimum delay to a network of VHF receiving stations on the ground for further distribution to interested observatories. The LOFT burst alert system will distribute the location of a transient event with $\sim 1$ arc minute accuracy to end users within $30 \mathrm{~s}$ (goal $20 \mathrm{~s}$ ) of the onboard detection of the burst in at least $2 / 3$ of the cases.

For a coded mask instrument, the deconvolution of the detector image into a sky image is computationally very demanding. The deconvolution will be done onboard by cross correlation of the mask pattern with the background subtracted detector image by discrete Fast Fourier Transform (DFFT) algorithm. The position is initially defined relative to the camera coordinate system, but is then, based on the pointing information, transformed into a position on the sky, which is compared with a catalog of known X-ray sources. If the position does not correspond to a known source and the calculations meet a certain set of quality/reliability criteria the software will send a short message with brief information about the event to the onboard data handling in order for it to be transmitted immediately to the ground via the spacecraft VHF transmitter system. The message will contain information on burst time, burst location, duration, and a set of quality flags for the use of the ground based users.

The LOFT burst alert system ground segment will be based on the network based on the equatorial subset of the VHF stations planned for the SVOM mission, or a similar dedicated network, in case the SVOM network will not be realized. The typical data rate for this system is $600 \mathrm{bits} / \mathrm{s}$, which allows a short message containing the basic burst information, to be transmitted in less than 2 seconds. The requirements of a maximum delay between the burst trigger and the delivery of the burst information packet of $<30 \mathrm{~s}$ is very conservative. A realistic goal will be a delay less than $20 \mathrm{~s}$, and we expect the system in many cases performing significantly better. The number of VHF ground stations needed to ensure continuous coverage is limited to 12 ideally placed stations However, the placement of the stations will eventually be determined by available land based sites with sufficient infrastructure (see Fig. 4). The VHF ground station will be managed by a central LOFT Alert Center, having the responsibility of validating the burst alerts and distributing the alerts to the end users.

We note that the LOFT mission does not include any capability for the satellite to do automated reorientations to observe the GRB afterglows with the LAD, as this would impose significant and costly requirements on the spacecraft autonomy. 


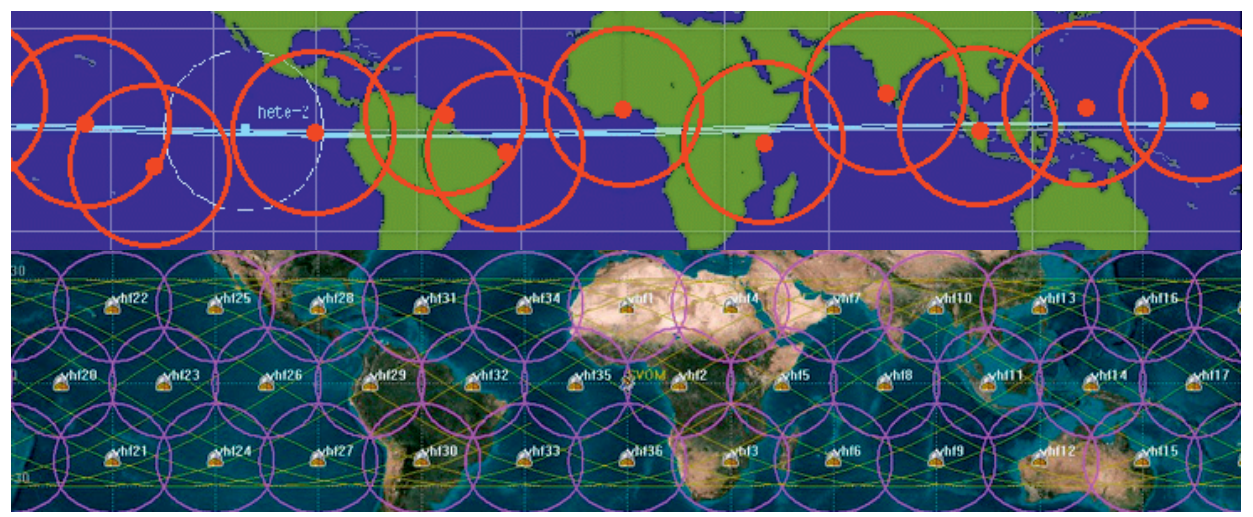

Fig. 4. The location of VHF stations for the former GRB mission HETE-II (top) and the theoretical SVOM network (bottom). The ideal LOFT burst alert system network consists of the 12 central/equatorial stations of the planned SVOM system.

\section{Conclusion}

Although LOFT is not designed to be a GRB mission, the WFM is expected to provide significant, independent contributions to the field of the gamma ray burst studies through the near real time burst alert system.

According to the current ESA selection schedule, it will be known by early 2014, if LOFT will move ahead from the assessment phase and be implemented for an expected launch in 2024.

\section{References}

[1] Feroci, M., Stella, L., van der Klis, M., et al., 2012, Exper. Astron., 34, 415

[2] Feroci, M., den Herder, J.W., Bozzi, E., et al., 2012, SPIE Conf. Ser., 8443-85

[3] "Cosmic Vision: Space Science for Europe 2015-2025", ESA Brochure, Vol. BR-247, 1-111 (15 Oct. 2005)

[4] Zane, S., Walton, D., Kennedy, T., et al., 2012, SPIE Conf. Ser., 8443-87

[5] Brandt, S., Hernanz, M., Alvarez, L., et al., 2012, SPIE Conf. Ser., 8443-88 
